\title{
review
}

\section{Selection of non-small cell lung cancer patients for intercalated chemotherapy and tyrosine kinase inhibitors}

\author{
Matjaz Zwitter ${ }^{1,2}$, Antonio Rossi ${ }^{3}$, Massimo Di Maio $^{4}$, Maja Pohar Perme ${ }^{5}$, Gilberto Lopes ${ }^{6}$ \\ ${ }^{1}$ Institute of Oncology Ljubljana, Ljubljana, Slovenia \\ ${ }^{2}$ Faculty of Medicine, University of Maribor, Maribor, Slovenia \\ ${ }^{3}$ Division of Medical Oncology, IRCCS Casa Sollievo della Sofferenza Hospital, San Giovanni Rotondo (FG), Italy \\ ${ }^{4}$ Division of Medical Oncology, Mauriziano Hospital, Oncology Department, University of Turin, Torino, Italy \\ ${ }^{5}$ Institute for Biostatistics and Medical Informatics, Faculty of Medicine, University of Ljubljana, Ljubljana, Slovenia \\ ${ }^{6}$ Centro Paulista de Oncologia e HCor Onco, members of the Oncoclínicas do Brasil Group, Sao Paulo, Brazil and Sylvester \\ Comprehensive Cancer Center, University of Miami, Miami, FL, USA
}

Radiol Oncol 2017; 51(3): 241-251.

Received 3 May 2017

Accepted 9 June 2017

Correspondence to: Prof. Matjaž Zwitter M.D., Ph.D., Department of Radiation Oncology, Institute of Oncology, Zaloška 2, SI-1000 Ljubljana, Slovenia. E-mail: matjaz.zwitter@guest.arnes.si

Disclosure: MZ has received honoraria for lectures from Roche, AstraZeneca and Merck Soreno and consultancy fee from Boehringer Ingelheim. AR has received honoraria for lectures from Roche, and acted as consultant for AstraZeneca, Roche, and Eli-Lilly. MDM acted as consultant for AstraZeneca, Boehringer Ingelheim, Eli Lilly, Bayer, Merck Sharp \& Dohme and Bristol Myers Squibb. MPP and GL did not report any conflict of interest.

Background. When treating patients with advanced non-small cell lung cancer (NSCLC) with tyrosine kinase inhibitors and chemotherapy, intercalated schedule with time separation between the two classes of drugs should avoid their mutual antagonism. In a survey of published trials, we focus on relation between eligibility criteria and effectiveness of intercalated treatment.

Methods. Published documents were identified using major medical databases, conference proceedings and references of published trials. Median progression-free survival (PFS) was taken as the basic parameter of treatment efficacy. Correlation between characteristics of patients and median PFS was assessed through the Pearson's correlation coefficient and the coefficient of determination, separately for first-line and second-line setting.

Results. The series includes 11 single-arm trials and 18 randomized phase II or phase III trials with a total of 2903 patients. Treatment-naive patients or those in progression after first-line treatment were included in 16 and 13 trials, respectively. In 14 trials, only patients with non-squamous histology were eligible. Proportion of patients with nonsquamous carcinoma (in first-line setting), proportion of never-smokers (both in first- and second-line setting) and proportion of epidermal growth factor receptor (EGFR) mutant patients (both in first- and second-line setting) showed a moderate or strong correlation with median PFS. In six trials of intercalated treatment applied to treatment-naive EGFR-mutant patients, objective response was confirmed in $83.1 \%$ of cases and median PFS was 18.6 months.

Conclusions. Most suitable candidates for intercalated treatment are treatment-naive patients with EGFR-mutant tumors, as determined from biopsy or liquid biopsy. For these patients, experience with intercalated treatment is most promising and randomized trials with comparison to the best standard treatment are warranted.

Key words: NSCLC; intercalated treatment; EGFR; tyrosine-kinase inhibitors

\section{Introduction}

Soon after the discovery of activating mutations of epidermal growth factor receptor (EGFR) and of their crucial role in determining sensitivity to tyrosine kinase inhibitors (TKIs), several trials confirmed the advantage of TKIs in comparison to chemotherapy for patients with advanced non- 
small cell lung cancer (NSCLC) with activating EGFR mutations. ${ }^{1-5}$ Due to higher response rate, longer time to progression and less toxicity, monotherapy with TKIs has been approved as the preferred first-line treatment for these patients. TKIs clearly improve short-term prognosis of patients with EGFR mutant lung cancer. Still, after a median interval of around one year, patients on treatment with TKIs develop resistance and experience disease progression. ${ }^{6}$ New treatments, either as first-line or at progression are needed. ${ }^{7}$

After huge disappointment due to four large negative trials of chemotherapy alone versus chemotherapy combined with continuous application of gefitinib or erlotinib ${ }^{8-11}$, the idea of combining TKIs with chemotherapy never fully recovered. Many research groups simply concluded that the two categories of drugs should not be used together and most of current pre-clinical and clinical research focuses on new drugs designed to overcome primary or acquired resistance to TKIs. ${ }^{12-14}$ The fact that the four trials were unselected regarding histology and smoking status received little attention. For a quarter of patients in the TRIBUTE trial, the status of EGFR mutations was later determined: when treated with the combination in comparison to chemotherapy alone, EGFR mutant patients had higher response rate and a trend towards longer progression-free survival (PFS), but no advantage in overall survival (OS). ${ }^{15}$

In spite of prevailing disappointment, some researchers insisted that the negative message of the four large trials should be accepted as new knowledge rather than ignored and developed the concept of intercalated treatment. The reasoning was clear: when applied concomitantly with chemotherapy, TKIs induce G1-phase cell-cycle arrest, due to which cell-cycle dependent chemotherapeutic agents will not be effective. ${ }^{16}$ Chemorefractoriness of cells harboring sensitizing-EGFR mutations in the presence of gefitinib was confirmed in vitro. ${ }^{17}$ Time separation with an interval of 6 days without TKIs to restore chemosensitivity of tumor cells should remove this mutual antagonism, so as to benefit from the efficacy of both classes of drugs..$^{18}$ Additional support for the concept of intercalated treatment came from laboratory experiments and from a clinical trial of sequential application of chemotherapy and TKI: proper sequence starting with cytotoxic drugs, followed by TKIs leads to their synergistic activity. ${ }^{19-23}$

So far, a few dozen clinical trials on intercalated treatment with chemotherapy and TKIs have been published. A recent review focused on trials with randomization between intercalated schedules and chemotherapy alone. ${ }^{24}$ While such a review is definitively valuable, a reader is confused when facing a list of trials with a wide range of eligibility criteria, leading to diverging conclusions. This review paper has a different goal. We believe that before proceeding with further clinical research, we should understand which patients will most likely benefit from intercalated treatment. Only after defining the optimal target $\mathrm{p}$ opulation, future trials can be designed to compare intercalated treatment to the best standard treatment in those selected patients.

\section{Methods}

\section{Selection of publications for analysis}

Published papers and conference $r$ eports on intercalated therapy for NSCLC were eligible for review. Intercalated treatment was defined as a treatment with cytotoxic drug(s), combined with an EGFR-TKI during a part of a treatment cycle. Trials on chemotherapy combined with continuous application of TKIs or a gap of less than 4 days, and trials using alternating cycles of cytotoxic drugs and of TKIs were not included in this review.

Pubmed was used to search for published trials on intercalated treatment for NSCLC, with the following descriptors and limits: NSCLC; chemotherapy and (erlotinib and/or gefitinib and/ or afatinib); clinical trial; 2006-2016 as the publication period; humans; English language. This initial search led to 686 publications. The list was then manually reviewed. Additional publications were identified using Web of Science cross-citation database, proceedings of major conferences during the past 4 years (IASLC World Lung Cancer Conference, ASCO, ESMO, European Lung Cancer Conference) and the list of references in published trials.

\section{Analysis of published experience}

Selected papers were analyzed, with focus on selection criteria and parameters of efficacy. Information on the proportion of patients with EGFR mutant tumors was often incomplete or missing. If not available in the text, an estimate on the proportion of patients with EGFR mutant disease was made. As the first step, all patients with squamous histology were considered as EGFR wild-type (EGFR wt). In case of non-squamous histology, an estimate was based on proportion of never-smokers vs. smokers 
and on ethnicity, using published meta-analysis on global pattern of EGFR mutations..$^{25}$

Due to the wide spectrum of eligibility criteria and of comparator arms, randomized trials were not considered an appropriate basis for analysis of efficacy of the intercalated treatment. We therefore chose a different approach. Experience from singlearm phase II trials and from the intercalated arm of randomized trials was analyzed in relation to histology, smoking status and EGFR mutation status, separately for treatment-naive and pre-treated patients. Median PFS was taken as a conventional indicator of efficacy.

\section{Statistical analysis}

Statistical analysis was limited to intercalated treatment, either in non-randomised trials or in the intercalated arm of randomised trials. The strength of correlation between proportion of non-squamous tumors, proportion of non-smokers and proportion of EGFR-mutation positive cases with median PFS was evaluated calculating the Pearson's correlation coefficient $(\mathrm{R})$ and the coefficient of determination (R-squared; R2). Pearson's R is a simple measure of the linear correlation between two variables, giving a value between +1 and -1 , where +1 is a total positive correlation, 0 is the absence of correlation, and -1 is a total negative correlation. The coefficient of determination is such that $0 \leq R 2 \leq 1$ : although there are no specific cut-offs to define a moderate, or a strong correlation, a higher R2 score indicates a stronger association.

Correlations were graphically described by bubble plots, where each bubble represents a study, with bubble size proportional to the number of patients included in each study. As all the analyses were weighted by sample size of each study, weighted least-squares regression line was calculated and reported in each graph.

Statistical analyses were conducted using SPlus (S-PLUS 6.0 Professional, release 1; Insightful Corporation, Seattle, WA, USA). Graphs were realized using SigmaPlot (Systat Software, San Jose, CA).

\section{Results}

\section{Identification of publications for analysis}

After the initial search through PubMed, 96 reports were selected to which 4 trials found through conference proceedings and 6 trials identified by cross-citation database were added for a total of

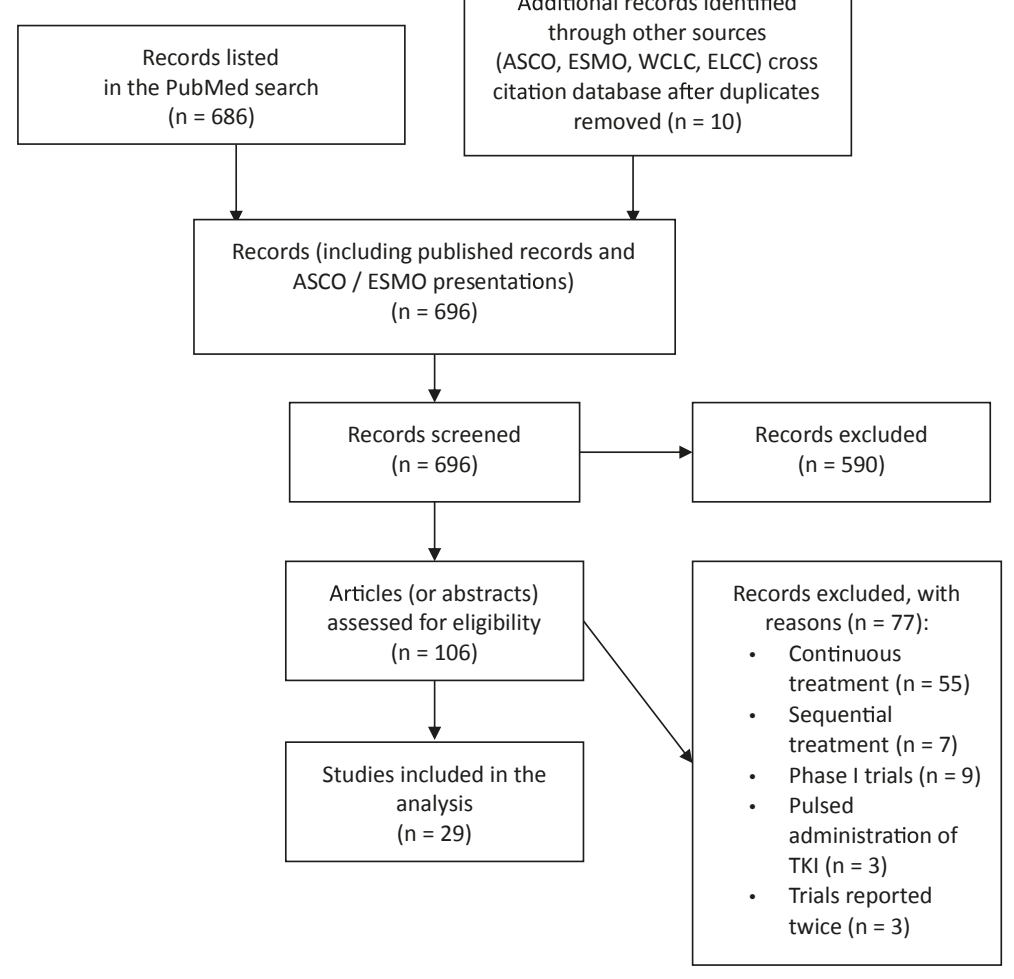

ASCO: American Society of Cli nical Oncology; ESMO: EuropeanSociety of Medical Oncology; WC LC : World Conference on Lung Cancer; EL CC : European Lung Cancer Co nference: TK I: Ty rosine kinase inhibitor

FIGURE 1. Flow diagram on selection of publications for analysis.

106 studies. A total of 29 trials were considered eligible for the analysis (Figure 1). The series includes 11 single-arm phase II trials, 16 randomized phase II trials and 2 phase III randomized trials, with a total of 2903 patients. ${ }^{26-54}$

\section{Eligibility criteria and treatments used}

Fourteen trials were limited to non-squamous histology, while 15 included patients with any histologic type of NSCLC. Sixteen trials were open only for treatment-naive patients, while 13 included those in progression after first-line treatment. The proportion of never-smokers was specified in all but two reports.

Five trials were limited to EGFR mutant disease and 4 to patients with EGFR wild-type or unknown tumors. Another 3 trials included a substantial proportion of EGFR mutant cases and separately reported the experience for this subpopulation of patients. Nine of the remaining 17 trials included information on EGFR status for some of their patients, but the proportion analyzed was usually low and thus inappropriate for any analysis. 
TABLE 1. Randomized trials on intercalated chemotherapy and TKIs for non-small cell lung cancer

\begin{tabular}{|c|c|c|c|c|c|c|c|c|c|}
\hline REFERENCE & TYPE OF TRIAL & $\begin{array}{l}\# \\
\text { OF } \\
\text { PTS }\end{array}$ & $\begin{array}{l}\text { SELECTION OF } \\
\text { PATIENTS }\end{array}$ & TREATMENT REGIMEN(s) & $\begin{array}{l}\% \text { never- } \\
\text { smokers }\end{array}$ & $\begin{array}{l}\text { \% EGFR } \\
\text { mutant, } \\
\text { intercalated } \\
\text { arm only }\end{array}$ & ORR (\%) & $\begin{array}{l}\text { MEDIAN PFS } \\
\text { (months) }\end{array}$ & $\begin{array}{l}\text { MEDIAN OS } \\
\text { (months) }\end{array}$ \\
\hline \multirow{2}{*}{$\begin{array}{l}\text { Mok } 2009 \\
\text { (FASTACT) }\end{array}$} & \multirow{2}{*}{$\begin{array}{l}\text { Randomized } \\
\text { Phase } 2\end{array}$} & 154 & $\begin{array}{l}\text { All histologies, } \\
\text { previously untreated }\end{array}$ & $\begin{array}{l}\text { Erlotinib, d 15-28 } \\
\text { Cycle q } 4 \text { weeks } \\
\text { Arm B (78 pts): } \\
\text { as above, placebo } \\
\text { instead of Erlotinib }\end{array}$ & $34 \%$ & $28 \%$ & $\begin{array}{l}\text { Arm A: } 35.5 \% \\
\text { Arm B: } 24.4 \%\end{array}$ & $\begin{array}{l}\text { Arm A: } 6.9 \mathrm{~m} \\
\text { Arm B: } 5.5 \mathrm{~m}\end{array}$ & $\begin{array}{l}\text { Arm A: } 17.3 \mathrm{~m} \\
\text { Arm B: } 17.7 \mathrm{~m}\end{array}$ \\
\hline & & 52 & $\begin{array}{l}\text { As above, } \\
\text { neversmokers }\end{array}$ & $\begin{array}{l}\text { Arm A (24 pts) } \\
\text { Arm B (28 pts) } \\
\text { Treatment as above }\end{array}$ & $100 \%$ & $49 \%$ & $\begin{array}{l}\text { Arm A: } 45.8 \% \\
\text { Arm B: } 32.1 \% \\
\text { P: not } \\
\text { reported }\end{array}$ & $\begin{array}{l}\text { Arm A: } 11.1 \mathrm{~m} \\
\text { Arm B: } 6.4 \mathrm{~m} \\
P=0.002\end{array}$ & Not reached \\
\hline Hirsch $2011^{27}$ & $\begin{array}{l}\text { Randomized } \\
\text { Phase } 2\end{array}$ & 143 & $\begin{array}{l}\text { Positive for EGFR } \\
\text { protein expression } \\
\text { and/or with high }\end{array}$ & $\begin{array}{l}\text { Arm A (71 pts): } \\
\text { Pacli d } 1 \\
\text { Carbo d } 1\end{array}$ & $28 \%$ & $10 \%$ & $\begin{array}{l}\text { Arm A: } 22.4 \% \\
\text { Arm B: } 11.6 \%\end{array}$ & $\begin{array}{l}\text { Arm A: } 4.6 \mathrm{~m} \\
\text { Arm B: } 2.7 \mathrm{~m}\end{array}$ & $\begin{array}{l}\text { Arm A: } 11.4 \mathrm{~m} \\
\text { Arm B: } 16.7 \mathrm{~m}\end{array}$ \\
\hline \multirow[t]{2}{*}{$\begin{array}{l}\text { Aerts } 2012 \\
\text { (NVALT 10) } 28\end{array}$} & \multirow[t]{2}{*}{$\begin{array}{l}\text { Randomized } \\
\text { Phase } 2\end{array}$} & \multirow[t]{2}{*}{231} & \multirow{2}{*}{$\begin{array}{l}\text { All histologies } \\
\text { Progression after } \\
\text { platin-based } \\
\text { chemotherapy }\end{array}$} & $\begin{array}{l}\text { Arm A (115 pts): } \\
\text { Erlotinib } \\
\text { Arm B (116 pts): }\end{array}$ & \multirow[t]{2}{*}{$7 \%$} & \multirow[t]{2}{*}{$4 \%$} & $\begin{array}{l}\text { Arm A: } 7 \% \\
\text { Arm B: } 13 \%\end{array}$ & $\begin{array}{l}\text { Arm A: } 4.9 \mathrm{~m} \\
\text { Arm B: } 6.1 \mathrm{~m}\end{array}$ & $\begin{array}{l}\text { Arm A: } 5.5 \mathrm{~m} \\
\text { Arm B: } 7.8 \mathrm{~m}\end{array}$ \\
\hline & & & & $\begin{array}{l}\text { Doce or Pem, d } 1 \\
\text { Erlotinib, d } 2-16 \\
\text { Cycle q } 3 \text { weeks }\end{array}$ & & & $P=0.03$ & $P=0.11$ & $P=0.01$ \\
\hline \multirow[t]{2}{*}{ Lee $2013^{29}$} & \multirow[t]{2}{*}{$\begin{array}{l}\text { Randomized } \\
\text { Phase } 2\end{array}$} & \multirow[t]{2}{*}{240} & \multirow[t]{2}{*}{$\begin{array}{l}\text { Non-squamous, } \\
\text { never-smokers, } \\
\text { Progression after } 1^{\text {st }} \\
\text { line chemotherapy }\end{array}$} & $\begin{array}{l}\text { Arm A (78 pts): } \\
\text { Pem d 1 } \\
\text { Erlotinib d } 2 \text { - 14, Cycle } \\
\text { q } 3 \text { weeks }\end{array}$ & \multirow[t]{2}{*}{$100 \%$} & \multirow[t]{2}{*}{$56 \%$} & $\begin{array}{l}\text { Arm A: } 44.7 \% \\
\text { Arm B: } 29.3 \% \\
\text { Arm C: } 10.0 \%\end{array}$ & $\begin{array}{l}\text { Arm A: } 7.4 \mathrm{~m} \\
\text { Arm B: } 3.8 \mathrm{~m} \\
\text { Arm C: } 4.4 \mathrm{~m}\end{array}$ & \multirow[t]{2}{*}{$\begin{array}{l}\text { Arm A: } 20.5 \mathrm{~m} \\
\text { Arm B: } 22.8 \mathrm{~m} \\
\text { Arm C: } 17.7 \\
\mathrm{~m}\end{array}$} \\
\hline & & & & $\begin{array}{l}\text { Arm B (82 pts): } \\
\text { Erlotinib continuously } \\
\text { Arm C ( } 80 \text { pts): } \\
\text { Pem d 1,Cycle q } 3 \\
\text { weeks }\end{array}$ & & & $P=0.001$ & $P=0.003$ & \\
\hline $\begin{array}{l}\text { Wu Y-L 2013 } \\
\text { (FASTACT 2) }{ }^{30}\end{array}$ & $\begin{array}{l}\text { Randomized } \\
\text { Phase } 3\end{array}$ & 97 & $\begin{array}{l}\text { As above, subgroup } \\
\text { with activating EGFR } \\
\text { mutations }\end{array}$ & & & & $P<0.0001$ & $P<0.0001$ & $P=0.009$ \\
\hline Auliac $2014{ }^{31}$ & $\begin{array}{l}\text { Randomised } \\
\text { Phase } 2\end{array}$ & 147 & $\begin{array}{l}\text { EGFR wild-type or } \\
\text { unknown } \\
\text { Progression after } \text { lst }^{\text {st }}\end{array}$ & $\begin{array}{l}\text { Arm A (73 pts): } \\
\text { Doce, d } 1 \\
\text { Erlotinib, d 2-16 }\end{array}$ & $7.5 \%$ & $4 \%$ & $\begin{array}{l}\text { Arm A: } 4.4 \% \\
\text { Arm B: } 1.4 \%\end{array}$ & $\begin{array}{l}\text { Arm A: } 2.2 \mathrm{~m} \\
\text { Arm B: } 2.5 \mathrm{~m}\end{array}$ & $\begin{array}{l}\text { Arm A: } 6.5 \mathrm{~m} \\
\text { Arm B: } 8.3 \mathrm{~m}\end{array}$ \\
\hline & & & line chemotherapy & $\begin{array}{l}\text { Cycle q } 3 \text { weeks } \\
\text { Arm B (74 pts): } \\
\text { Doce, d l }\end{array}$ & & & $P=n s$ & $P=n s$ & $P=n s$ \\
\hline $\begin{array}{l}\text { Karavasilis } \\
2014^{32}\end{array}$ & $\begin{array}{l}\text { Randomized } \\
\text { Phase } 2\end{array}$ & 50 & $\begin{array}{l}\text { All histologies } \\
\text { Previously untreated }\end{array}$ & $\begin{array}{l}\text { Arm A (25 pts): } \\
\text { Doce, d 1 } \\
\text { Erlotinib, d } 9-20 \\
\text { Arm B (25 pts): } \\
\text { Doce, d l } \\
\text { Erlotinib, d } 3-14 \\
\text { Cycle q } 3 \text { weeks }\end{array}$ & $10 \%$ & $11 \%$ & $\begin{array}{l}\text { Arm A: } 24 \% \\
\text { Arm B: } 12 \%\end{array}$ & $\begin{array}{l}\text { Arm A: } 2.9 \mathrm{~m} \\
\text { Arm B: } 4.2 \mathrm{~m}\end{array}$ & $\begin{array}{l}\text { Arm A: } 9.9 \mathrm{~m} \\
\text { Arm B: } 10.8 \mathrm{~m}\end{array}$ \\
\hline Mok $2014{ }^{33}$ & $\begin{array}{l}\text { Randomized } \\
\text { Phase } 2\end{array}$ & 123 & $\begin{array}{l}\text { Unselected, } \\
\text { progression after } \\
\text { platin-based ChT }\end{array}$ & $\begin{array}{l}\text { Arm A (63 pts): } \\
\text { Eribulin mesylate, dl } \\
\text { Erlotinib, d 2-16 }\end{array}$ & $24 \%$ & $28 \%$ & $\begin{array}{l}\text { Arm A: } 13 \% \\
\text { Arm B: } 17 \%\end{array}$ & $\begin{array}{l}\text { Arm A: } 3.5 \mathrm{~m} \\
\text { Arm B: } 3.8 \mathrm{~m}\end{array}$ & $\begin{array}{l}\text { Arm A: } 7.6 \mathrm{~m} \\
\text { Arm B: } 8.5 \mathrm{~m}\end{array}$ \\
\hline & & & & $\begin{array}{l}\text { Cycle q } 3 \text { weeks } \\
\text { Arm B (60 pts): } \\
\text { Eribulin mesylate, d } 1 \\
\text { and } 8 \\
\text { Erlotinib, d 15-28 } \\
\text { Cycle q } 4 \text { weeks }\end{array}$ & & & $P=n s$ & $P=n s$ & $P=n s$ \\
\hline
\end{tabular}




\begin{tabular}{|c|c|c|c|c|c|c|c|c|c|}
\hline REFERENCE & TYPE OF TRIAL & $\begin{array}{l}\# \\
\text { OF } \\
\text { PTS }\end{array}$ & $\begin{array}{l}\text { SELECTION OF } \\
\text { PATIENTS }\end{array}$ & TREATMENT REGIMEN(s) & $\begin{array}{l}\text { \% never- } \\
\text { smokers }\end{array}$ & $\begin{array}{l}\text { \% EGFR } \\
\text { mutant, } \\
\text { intercalated } \\
\text { arm only }\end{array}$ & ORR (\%) & $\begin{array}{l}\text { MEDIAN PFS } \\
\text { (months) }\end{array}$ & $\begin{array}{l}\text { MEDIAN OS } \\
\text { (months) }\end{array}$ \\
\hline \multirow[t]{2}{*}{ Yu $2014^{34}$} & $\begin{array}{l}\text { Randomized } \\
\text { Phase } 2\end{array}$ & 117 & $\begin{array}{l}\text { Non-squamous, } \\
\text { previously untreated }\end{array}$ & $\begin{array}{l}\text { Arm A (58 pts): } \\
\text { Pem, d 1 } \\
\text { Cis or Carbo, d 1 } \\
\text { Gefitinib, d } 3-16 \\
\text { Cycle q } 3 \text { weeks } \\
\text { Arm B (57 pts): } \\
\text { As above, no Gefitinib }\end{array}$ & $58 \%$ & $40 \%$ & $\begin{array}{l}\text { Arm A: } 50.0 \% \\
\text { Arm B: } 47.7 \% \\
P=n s\end{array}$ & $\begin{array}{l}\text { Arm A: } 7.9 \mathrm{~m} \\
\text { Arm B: } 7.0 \mathrm{~m} \\
P=n s\end{array}$ & $\begin{array}{l}\text { Arm A: } 25.4 \\
m \\
\text { Arm B: } 20.8 \mathrm{~m} \\
P=n s\end{array}$ \\
\hline & & 32 & $\begin{array}{l}\text { As above, subgroup } \\
\text { with activating EGFR } \\
\text { mutations }\end{array}$ & $\begin{array}{l}\text { Arm A: } 14 \text { pts } \\
\text { Arm B: } 18 \text { pts } \\
\text { Treatment as above }\end{array}$ & $\begin{array}{l}\text { Not } \\
\text { separately } \\
\text { reported }\end{array}$ & $100 \%$ & $\begin{array}{l}\text { Arm A: } 76.9 \% \\
\text { Arm B: } 50.0 \% \\
P=0.13\end{array}$ & $\begin{array}{l}\text { Arm A: } \\
\text { Not reached } \\
\text { Arm B: } 14.0 \mathrm{~m} \\
P=0.017\end{array}$ & Not reached \\
\hline Choi $2015^{35}$ & $\begin{array}{l}\text { Randomized } \\
\text { Phase } 2\end{array}$ & 90 & $\begin{array}{l}\text { NSCLC, EGFR wild. } \\
\text { type or unknown, } \\
\text { PS } 0-2 \text {, previously } \\
\text { untreated }\end{array}$ & $\begin{array}{l}\text { Arm A (44 pts): } \\
\text { Pem, d l } \\
\text { Carbo, d } 1 \\
\text { Gefitinib, d } 2 \text { - } 15 \\
\text { Cycle q } 3 \text { weeks x } 4 \\
\text { Maintenance Gefitinib } \\
\text { Arm B (46 pts): } \\
\text { As Arm A, no Gefitinib }\end{array}$ & $10 \%$ & $10 \%$ & $\begin{array}{l}\text { Arm A: } 41.9 \% \\
\text { Arm B: } 39.5 \% \\
P=n s\end{array}$ & $\begin{array}{l}\text { Arm A: } 4.1 \mathrm{~m} \\
\text { Arm B: } 4.1 \mathrm{~m} \\
P=n s\end{array}$ & $\begin{array}{l}\text { Arm A: } 9.3 \mathrm{~m} \\
\text { Arm B: } 10.5 \mathrm{~m} \\
P=n s\end{array}$ \\
\hline Juan $2015^{36}$ & $\begin{array}{l}\text { Randomized } \\
\text { Phase } 2\end{array}$ & 68 & $\begin{array}{l}\text { All histologies } \\
\text { Progression after } \\
\text { platin-based } \\
\text { chemotherapy }\end{array}$ & $\begin{array}{l}\text { Arm A ( } 33 \text { pts): } \\
\text { Doce q } 3 \text { weeks } \\
\text { Erlotnib, d } 2-16 \\
\text { Arm B ( } 35 \text { pts): } \\
\text { Erlotinib continuously }\end{array}$ & $6 \%$ & $5 \%$ & $\begin{array}{l}\text { Arm A: } 3 \% \\
\text { Arm B: } 9 \% \\
P=0.19\end{array}$ & $\begin{array}{l}\text { Arm A: } 3.0 \mathrm{~m} \\
\text { Arm B: } 2.1 \mathrm{~m} \\
P=0.09\end{array}$ & $\begin{array}{l}\text { Arm A: } 7.5 \mathrm{~m} \\
\text { Arm B: } 5.2 \mathrm{~m} \\
P=0.19\end{array}$ \\
\hline Lu $2015^{37}$ & $\begin{array}{l}\text { Randomized } \\
\text { Phase } 3\end{array}$ & 219 & $\begin{array}{l}\text { Adenocarcinoma, } \\
\text { EGFR unknown, } \\
\text { non-smokers, no } \\
\text { progression after } 2 \\
\text { cycles of gem-carbo }\end{array}$ & $\begin{array}{l}\text { Arm A ( } 109 \text { pts): } \\
\text { Gem, d } 1 \text { and } 8 \\
\text { Carbo, d } 1 \\
\text { Gefitinib d } 15-25 \text { and } \\
\text { maintenance } \\
\text { Cycle q } 4 \text { weeks x } 4 \\
\text { Arm B (110 pts): } \\
\text { As above, no Gefitinib }\end{array}$ & $100 \%$ & $72 \%$ & Not reported & $\begin{array}{l}\text { Arm A: } 10 \mathrm{~m} \\
\text { Arm B: } 4.4 \mathrm{~m} \\
P=0.001\end{array}$ & Not reported \\
\hline $\begin{array}{l}\text { Michael } \\
2015^{38}\end{array}$ & $\begin{array}{l}\text { Randomized } \\
\text { Phase } 2\end{array}$ & 54 & $\begin{array}{l}\text { All histologies } \\
\text { PS } 2 \text { or elderly } \\
\text { Previously untreated }\end{array}$ & $\begin{array}{l}\text { Arm A (28 pts): } \\
\text { Gem d l and } 8 \\
\text { Erlotinib days } 15-28 \\
\text { Cycle q } 4 \text { weeks } \\
\text { Arm B ( } 26 \text { pts): } \\
\text { Gem dl and } 8 \\
\text { Cycle q } 4 \text { weeks }\end{array}$ & $15 \%$ & $12 \%$ & $\begin{array}{l}\text { Arm A: } 6 \% \\
\text { Arm B: } 23 \% \\
\text { P: ns }\end{array}$ & $\begin{array}{l}\text { Arm A: } 2.5 \mathrm{~m} \\
\text { Arm B: } 1.9 \mathrm{~m} \\
\text { P: ns }\end{array}$ & $\begin{array}{l}\text { Arm A: } 3.9 \text { m } \\
\text { Arm B: } 4.4 \text { m } \\
\text { P: ns }\end{array}$ \\
\hline Han $2016{ }^{39}$ & $\begin{array}{l}\text { Randomized } \\
\text { Phase } 2\end{array}$ & 121 & $\begin{array}{l}\text { Adenocarcinoma, } \\
\text { EGFR mutant, } \\
\text { previously untreated }\end{array}$ & $\begin{array}{l}\text { Arm A ( } 40 \text { pts): } \\
\text { Pem, dl + } \\
\text { maintenance } \\
\text { Carbo, dl for } \leq 6 \text { cycles } \\
\text { Gefitinib, d } 5-21+ \\
\text { maintenance } \\
\text { Cycle q } 4 \text { weeks } \\
\text { Arm B ( } 40 \text { pts): } \\
\text { As above, no Gefitinib } \\
\text { Arm C (4l pts): } \\
\text { Gefitinib alone }\end{array}$ & $\begin{array}{l}\text { Not } \\
\text { reported }\end{array}$ & $100 \%$ & $\begin{array}{l}\text { Arm A: } 82.5 \% \\
\text { Arm B: } 32.5 \% \\
\text { Arm C: } 65.9 \% \\
\text { P: } 0.04\end{array}$ & $\begin{array}{l}\text { Arm A: } 18.8 \mathrm{~m} \\
\text { Arm B: } 5.7 \mathrm{~m} \\
\text { Arm C: } 12.0 \mathrm{~m} \\
\text { P: not } \\
\text { reported }\end{array}$ & Not reached \\
\hline $\begin{array}{l}\text { Lara } 2016 \\
\text { (SWOG } \\
\text { SO709) }\end{array}$ & $\begin{array}{l}\text { Randomized } \\
\text { Phase } 2\end{array}$ & 59 & $\begin{array}{l}\text { PS 2, Proteomics: } \\
\text { VeriStrat-good } \\
\text { status, previously } \\
\text { untreated }\end{array}$ & $\begin{array}{l}\text { Arm A ( } 33 \text { pts): } \\
\text { Erlotinib } \\
\text { Arm B ( } 26 \text { pts): } \\
\text { Pacli d } 1 \\
\text { Carbo d } 1 \\
\text { Erlotinib d } 2 \text { - } 16 \\
\text { Cycle q } 3 \text { weeks } \times 4 \\
\text { Maintenance Erlotinib }\end{array}$ & $20 \%$ & $20 \%$ & $\begin{array}{l}\text { Arm A: } 6 \% \\
\text { Arm B: } 23 \% \\
P=0.06\end{array}$ & $\begin{array}{l}\text { Arm A: } 1.6 \mathrm{~m} \\
\text { Arm B: } 4.6 \mathrm{~m} \\
P=0.06\end{array}$ & $\begin{array}{l}\text { Arm A: } 6.0 \mathrm{~m} \\
\text { Arm B: } 11.0 \mathrm{~m} \\
P=0.27\end{array}$ \\
\hline Li $2016^{41}$ & $\begin{array}{l}\text { Randomized } \\
\text { Phase } 2\end{array}$ & 79 & $\begin{array}{l}\text { Predominantly non- } \\
\text { squamous } \\
\text { Progression after } 1^{\text {st }} \\
\text { line chemotherapy }\end{array}$ & $\begin{array}{l}\text { Arm A ( } 27 \text { pts): } \\
\text { Pem d } 1 \\
\text { Cycle q } 3 \text { weeks } \\
\text { Arm B ( } 52 \text { pts): } \\
\text { Pem d } 1 \\
\text { Erlotinib d } 2 \text { - } 17 \\
\text { Cycle q } 3 \text { weeks }\end{array}$ & $\begin{array}{l}\text { Not } \\
\text { reported }\end{array}$ & $19 \%$ & $\begin{array}{l}\text { Arm A: } 10 \% \\
\text { Arm B: } 29 \% \\
P=0.17\end{array}$ & $\begin{array}{l}\text { Arm A: } 2.9 \mathrm{~m} \\
\text { Arm B: } 4.7 \mathrm{~m} \\
P=0.26\end{array}$ & $\begin{array}{l}\text { Arm A: } 8.3 \mathrm{~m} \\
\text { Arm B: } 9.7 \mathrm{~m} \\
P=0.28\end{array}$ \\
\hline Lee 201642 & $\begin{array}{l}\text { Randomized } \\
\text { Phase } 2\end{array}$ & 76 & $\begin{array}{l}\text { Adenocarcinoma, } \\
\text { neversmokers, } \\
\text { Previously untreated }\end{array}$ & $\begin{array}{l}\text { Arm A ( } 39 \text { pts): } \\
\text { Pem dl } \\
\text { Carbo d } 1 \\
\text { Gefitinib d } 5-18+ \\
\text { maintenance } \\
\text { Cycle q } 3 \text { weeks x } \\
\text { max } 9 \\
\text { Arm B ( } 37 \text { pts): } \\
\text { As arm A, placebo } \\
\text { instead of Gefitinib } \\
\text { At progression: Gefitinib } \\
\text { for arm B }\end{array}$ & $100 \%$ & $42 \%$ & $\begin{array}{l}\text { Arm A: } 79.5 \% \\
\text { Arm B: } 51.4 \% \\
P=0.01\end{array}$ & $\begin{array}{l}\text { Arm A: } 12.8 \mathrm{~m} \\
\text { Arm B: } 7.0 \mathrm{~m} \\
P=0.009\end{array}$ & $\begin{array}{l}\text { Arm A: } 29.2 \mathrm{~m} \\
\text { Arm B: } 20.4 \mathrm{~m} \\
P=0.15\end{array}$ \\
\hline
\end{tabular}




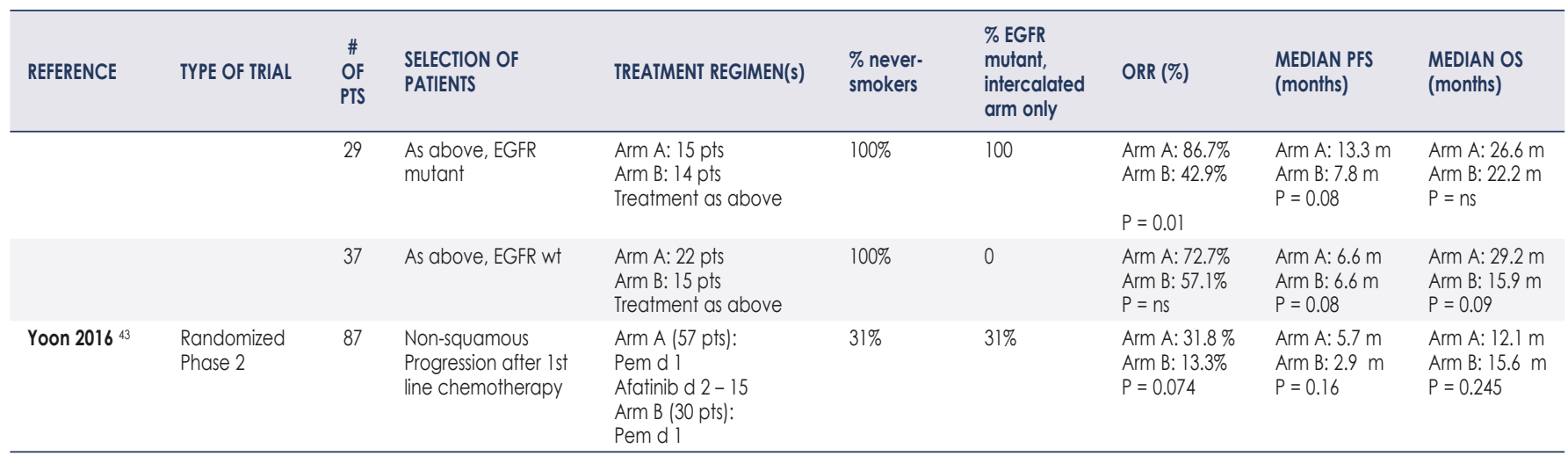

Carbo = carboplatin; $\mathrm{Cis}=$ cisplatin; Doce $=$ docetaxel; $\mathrm{Gem}=$ gemcitabine; ORR = overall response rate; OS = overall survival; Pacli $=$ paclitaxel; $\mathrm{PFS}=$ progression-free survival; Pem = pemetrexed; PTS = patient

Erlotinib and gefitinib were used in 17 and 9 trials, respectively. In 2 trials, either erlotinib or gefitinib was used, while afatinib was used in 1 trial.

Among 18 randomized trials (Table 1), chemotherapy alone was the comparator arm in 10 trials. Four trials used TKI alone as the comparator, 2 trials compared two different intercalated schedules, while 2 trials used 3-arm design with comparison among intercalated schedule, chemotherapy alone or TKIs alone. According to PFS as the most common parameter of efficacy, the intercalated schedule was superior to the comparator in 12 trials, crossing the conventional margin of $\mathrm{P}<0.05$ in 7 trials. No significant difference was seen in 4 other trials and in 2 trials comparing two different intercalated schedules of intercalated therapy.

Basic data on single-arm Phase 2 trials are presented in Table 2.

\section{Efficacy of intercalated treatment}

Figure 2 shows correlation between median PFS and proportion of patients with non-squamous histology (Panel A), proportion of never-smokers (Panel B) and proportion of EGFR mutant patients (Panel C).

As for tumor histology, there was a moderate correlation between proportion of non-squamous tumors and median PFS in the first-line setting $(\mathrm{R}=$ $0.61, R^{2}=0.38, p=0.02$ ). The slope of the regression line $(0.23)$ suggests that a $10 \%$ increase in the proportion of non-squamous tumors corresponds to a 2.3-months improvement in median PFS in first-line. On the other hand, the correlation was very weak in the second-line setting $\left(\mathrm{R}=0.09, R^{2}=0.01, p=0.77\right)$.

As for smoking status, there was a moderate correlation between proportion of non-smokers and median PFS, both in the first-line and in the second-line setting (first-line: $\mathrm{R}=0.55, R^{2}=0.30, p$ $=0.04$; second-line: $\left.\mathrm{R}=0.65, R^{2}=0.42, p=0.02\right)$. In detail, the slope of the regression line $(0.09$ for the first-line and 0.05 for the second-line) suggests that a $10 \%$ increase in the proportion of non-smokers corresponds to a 0.9-months improvement in median PFS in first-line, and to a 0.5-months improvement in median PFS in second-line.
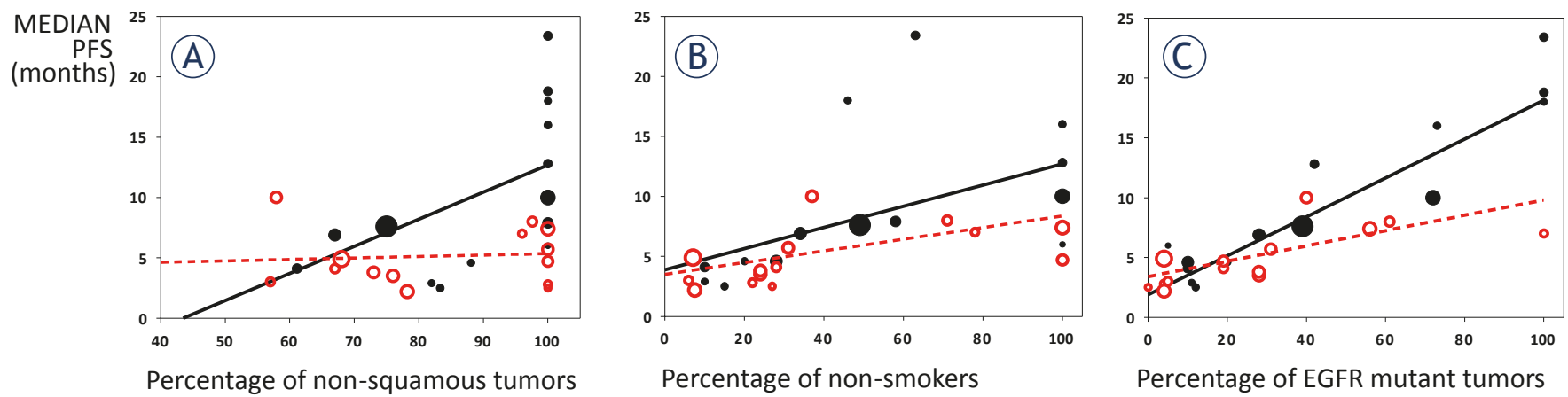

FIGURE 2. Correlation between median PFS and proportion of patients with non-squamous histology (A), proportion of never-smokers (B) and proportion of EGFR mutant patients $(C)$. Black solid marks and black solid lines are for $1^{\text {st }}$ line treatment; red hollow marks and red interrupted lines for $2^{\text {nd }}$ line treatment. Bubble size corresponds to the number of patients in a trial. 
TABLE 2. Single-arm Phase II trials on intercalated chemotherapy and TKls for non-small cell lung cancer

\begin{tabular}{|c|c|c|c|c|c|c|c|c|}
\hline REFERENCE & $\begin{array}{l}\# \\
\text { OF } \\
\text { PTS }\end{array}$ & $\begin{array}{l}\text { SELECTION OF } \\
\text { PATIENTS }\end{array}$ & TREATMENT REGIMEN(s) & $\begin{array}{l}\% \text { never- } \\
\text { smokers }\end{array}$ & \% EGFR mutant & ORR (\%) & MEDIAN PFS (months) & $\begin{array}{l}\text { MEDIAN OS } \\
\text { (months) }\end{array}$ \\
\hline Oshita $2010^{44}$ & 16 & $\begin{array}{l}\text { Unselected, } \\
\text { previously } \\
\text { untreated }\end{array}$ & $\begin{array}{l}\text { Pacli d 1 } \\
\text { Irino d 1 } \\
\text { Gefitinib d 8-14 } \\
\text { Cycle q } 3 \text { weeks }\end{array}$ & Not reported & $25 \%$ & $43.8 \%$ & Not reported & $18.1 \mathrm{~m}$ \\
\hline $\begin{array}{l}\text { Sangha } \\
201145\end{array}$ & 39 & $\begin{array}{l}\text { All histologies } \\
\text { Progression } \\
\text { after } 1^{\text {st line }} \\
\text { chemotherapy }\end{array}$ & $\begin{array}{l}\text { Doce d } 1 \\
\text { Erlotinib days } 2-16 \\
\text { Cycle q } 3 \text { weeks }\end{array}$ & $28 \%$ & $19 \%$ & $28.2 \%$ & $4.1 \mathrm{~m}$ & $18.2 \mathrm{~m}$ \\
\hline $\begin{array}{l}\text { Minami } \\
2013^{46}\end{array}$ & 27 & $\begin{array}{l}\text { Non-squamous } \\
\text { Progression } \\
\text { after } 1^{\text {st line }} \\
\text { chemotherapy }\end{array}$ & $\begin{array}{l}\text { Pem, d } 1 \\
\text { Erlotinib, d } 2-16 \\
\text { Cycle q } 3 \text { weeks }\end{array}$ & $22 \%$ & $4 \%$ & $11.1 \%$ & $2.8 \mathrm{~m}$ & $15.8 \mathrm{~m}$ \\
\hline $\begin{array}{l}\text { Yoshimura } \\
2013^{47}\end{array}$ & 27 & $\begin{array}{l}\text { Activating EGFR } \\
\text { mutations, } \\
\text { Progression after } \\
\text { TKI }\end{array}$ & $\begin{array}{l}\text { Pem, d } 1 \\
\text { Erlotinib or Gefitinib, days } \\
2-16 \\
\text { Cycle } q 3 \text { weeks }\end{array}$ & $78 \%$ & $100 \%$ & $25.9 \%$ & $7.0 \mathrm{~m}$ & $11.4 \mathrm{~m}$ \\
\hline Fang 201449 & 57 & $\begin{array}{l}\text { Unselected, } \\
\text { progression after } \\
\text { platin-based ChT }\end{array}$ & $\begin{array}{l}\text { Gem, } d l \text { and } 8 \\
\text { Cis, d l-3 } \\
\text { Gefitinib, } d 10-24 \\
\text { Cycle } q 4 \text { weeks }\end{array}$ & $37 \%$ & $40 \%$ & $11 \%$ & $10 \mathrm{~m}$ & $15.2 \mathrm{~m}$ \\
\hline Yang $2014^{50}$ & 29 & $\begin{array}{l}\text { Adenocarcinoma, } \\
\text { non-smokers, } \\
\text { EGFR unknown, } \\
\text { previously } \\
\text { untreated }\end{array}$ & $\begin{array}{l}\text { Pacli, dl } \\
\text { Carbo, dl } \\
\text { Gefitinib, d } 8-17 \text { + } \\
\text { maintenance } \\
\text { Cycle q } 3 \text { weeks }\end{array}$ & $100 \%$ & $73 \%$ & $74.1 \%$ & $16 \mathrm{~m}$ & Not reached \\
\hline Zwitter 2014 51 & 15 & $\begin{array}{l}\text { Adenocarcinoma, } \\
\text { light/never } \\
\text { smokers, EGFR } \\
\text { wild-type or } \\
\text { unknown } \\
\text { Previously } \\
\text { untreated }\end{array}$ & $\begin{array}{l}\text { Gem } d 1 \text { and } 4 \\
\text { Cis } d 2 \\
\text { Erlotinib } d 5-15 \\
\text { Cycle } q 3 \text { weeks } \times 4-6 \\
\text { Maintenance Erlotinib }\end{array}$ & $100 \%$ & $5 \%$ & $33 \%$ & $6.0 \mathrm{~m}$ & $7.6 \mathrm{~m}$ \\
\hline $\begin{array}{l}\text { Zwitter } 2016 \\
\text { (ITAC 2) }^{54}\end{array}$ & 38 & $\begin{array}{l}\text { Activating EGFR } \\
\text { mutations } \\
\text { Previously } \\
\text { untreated }\end{array}$ & $\begin{array}{l}\text { Gem } d 1 \text { and } 4 \\
\text { Cis } d 2 \\
\text { Erlotinib } d 5-15 \\
\text { Cycle } q 3 \text { weeks } \times 4-6 \\
\text { Maintenance Erlotinib }\end{array}$ & $63 \%$ & $100 \%$ & $84.2 \%$ & $23.4 \mathrm{~m}$ & $38.3 m$ \\
\hline
\end{tabular}

Carbo = carboplatin; $\mathrm{Cis}=$ cisplatin; Doce = docetaxel; Gem = gemcitabine; Irino = irinotecan; ORR = overall response rate; OS = overall survival; Pacli = paclitaxel; PFS = progression-free survival; Pem = pemetrexed; PTS = patient

As for EGFR mutational status, there was a strong correlation between proportion of EGFR mutation-positive tumors and median PFS, both in the first-line and in the second-line setting (firstline: $\mathrm{R}=0.91, R^{2}=0.83, p<0.0001$; second-line: $\mathrm{R}=$ $0.69, R^{2}=0.48, p=0.006$ ). The slope of the regression line $(0.16$ for the first-line and 0.06 for the secondline) suggests that a $10 \%$ increase in the proportion of EGFR mutation-positive tumors corresponds to a 1.6-months improvement in median PFS in firstline, and to a 0.6 -months improvement in median PFS in second-line.

Six trials on treatment-naive patients with EGFR mutant disease reported excellent response rate of $83.1 \%$ (range: $76.9 \%$ to $84.2 \%$ ). $30,34,39,42,52,54$ Five of these trials presented data on median PFS ranging from 13.3 to 23.4 months (median PFS for all 5 trials: 18.6 months). This figure does not include an 
additional trial from this category which reported $86 \%$ PFS at 15 months, with no data on median PFS due to relatively short follow-up. ${ }^{34}$

\section{Discussion}

Most surveys and meta-analyses focus on the question of efficacy and/or toxicity of a particular new treatment in comparison with the standard approach. This was also the case with intercalated treatment for advanced NSCLC. On the basis of published randomized trials, a recent meta-analysis concluded that intercalated treatment is superior to the comparator arm. ${ }^{24}$ While this meta-analysis offered a valuable insight into an area which is not in the main stream of current clinical research, the question of efficacy of intercalated treatment is too complex to be answered by a simple comparison.

A critical look at all published trials reveals great heterogeneity in eligibility criteria including treatment-naive patients or those in progression after first-line therapy. In addition, we see a whole spectrum of biologically divergent disease: all histologic types or only non-squamous histology; exclusively EGFR-mutant disease, only EGFR-wt tumors or, in most trials, both groups. On such a heterogeneous basis and without considering the optimal standard therapy for a particular population of patients, the value of a new approach cannot be assessed. Is the intercalated treatment superior to chemotherapy alone for EGFR-wt patients or superior to TKI alone for EGFR-mutant patients? To be more concrete: it comes as no surprise that intercalated treatment was superior to chemotherapy alone for a population which included a substantial proportion of EGFR-mutant patients; $26,29,30,34,37,39,42$ and superior to TKIs alone for a population of predominantly EGFR-wt patients. ${ }^{36,40}$ It is not the intercalated approach, but inclusion of an effective drug into the schedule which may be responsible for the positive experience in these trials. We believe that on the basis of randomized clinical trials published so far, the question of superiority of intercalated schedules over the standard treatment cannot be answered.

Our estimate on the proportion of EGFR mutant patients, as used in the analysis, includes a considerable degree of uncertainty. Only half of trials $(14 / 29)$ included information on the proportion of EGFR-mutant vs. EGFR-wt tumors for more than $50 \%$ of patients. Other trials reported results of EGFR analysis for a minority of patients, or (in 8 publications) no such information. We therefore made an estimate, based on histologic types of tumors (available for 28/29 trials), on proportion of never-smokers (available for 27/29 trials) and on the country where a trial was performed, using tables from a recent meta-analysis. ${ }^{25}$ This approach led us to the best possible estimate, but the results should nevertheless be regarded as exploratory and tentative. In future, precise molecular diagnostics should minimize these uncertainties.

Our survey does not include analysis of toxicity of different schedules of intercalated treatment. This aspect was clearly presented in a recent review: apart from the expected skin toxicity and diarrhea, intercalated schedules do not present a disproportional burden to patients. ${ }^{24}$

Relation between selection criteria and effectiveness of intercalated treatment is shown in Figure 2. On the basis of pooled data on median PFS from randomized and single-arm trials, it is clear that pre-treated patients are not good candidates for a treatment which includes a modality to which resistance has already developed. While the proportion of patients with non-squamous histology and of never-smokers determine efficacy of intercalated treatment, EGFR mutations are clearly the strongest predictive factor for longer PFS. By far the greatest benefit was for treatment-naive patients with activating EGFR mutations. According to trials in which this group of patients was reported separately, their median PFS after intercalated treatment ranged between 13.3 and 23.4 months; median PFS for the pooled data was 18.4 months. This figure is substantially above PFS of 9 to 13 months, as reported for TKIs as monotherapy for EGFR-mutant NSCLC. ${ }^{26,55-58}$ Response rate was also very high: $83.1 \%$, with a substantial proportion of complete remissions. Our survey is in accordance with a recent editorial and with a meta-analysis, which pointed to intercalated regimens as the most promising first-line treatment for EGFR- mutated NSCLC. ${ }^{59,60}$

With the introduction of liquid biopsy from peripheral blood, the category of patients with undetermined EGFR mutant status should be very small. ${ }^{61}$ In case this new technique is not available, previously untreated never-smokers with non-squamous histology for whom EGFR status cannot be determined should also be considered for trials testing the role of intercalated treatment. Depending on ethnicity, approximately half of them have EGFR mutant tumors, in which case addition of TKI to cytotoxic drugs would be clearly beneficial; the other half with EGFR negative tu- 
mors should benefit from cytotoxic drugs included in the intercalated schedule.

In future trials of intercalated treatment, pemetrexed with platin appears as the preferred option for the cytotoxic component. Regarding the choice of TKI, virtually all current experience is limited to erlotinib and gefitinib. Still, in view of recent very favorable experience for patients who developed resistance mutations, osimertinib intercalated with chemotherapy deserves to be considered either in first-line setting or for patients in progression after first-generation TKIs. ${ }^{62}$

In two recent phase II randomized trials, patients with treatment-naive advanced EGFR- mutant NSCLC were treated with continuous gefitinib in combination with chemotherapy. As the comparator arm, sequential gefitinib and chemotherapy or gefitinib alone were applied. ${ }^{63,64}$ In both trials, patients on the combination with continuous gefitinib and chemotherapy experienced longer PFS. These reports support the advantage of combined treatment with chemotherapy and TKIs and re-open the dilemma between their concomitant and intercalated application. Combination of TKIs with bevacizumab is another possibility which deserves further attention. ${ }^{65}$

We are perfectly aware that the correlation we have reported between the proportion of patients with EGFR mutation positive tumor and the median PFS obtained with the intercalated treatment could be reasonably observed also with EGFR inhibitors alone. The only way to assess the real added value of intercalated treatment is a randomized trial with comparison to TKI alone and overall survival as the principal endpoint.

In conclusion, intercalated treatment with cytotoxic drugs and TKIs is a promising approach for patients with previously untreated advanced NSCLC with activating EGFR mutations, as well as for never-smokers with adenocarcinoma and undetermined EGFR status. For these patients, randomized trials with comparison to the optimal standard treatment, or possibly to a third arm with continuous application of TKIs in combination with chemotherapy should define the preferred treatment approach.

\section{Funding}

This research did not receive any specific grant from funding agencies in the public, commercial, or not-for-profit sectors.

\section{Acknowledgements}

Marjeta Jerala and David Ožura from the Library of the Institute of Oncology Ljubljana provided valuable help in retrieving full-length papers for analysis. Sincere thanks to Lars and Dyanne Søraas for intellectual support and language editing.

\section{References}

1. Lynch TJ, Bell DW, Sordella R, Gurubhagavatula S, Okimoto RA, Brannigan $\mathrm{BW}$, et al. Activating mutations in the epidermal growth factor receptor underlying responsiveness of non-small-cell lung cancer to gefitinib. $N$ Engl J Med 2004; 350: 2129-39. doi:10.1056/NEJMoa040938

2. Mok TS, Wu YL, Thongprasert S, Yang CH, Chu DT, Saijo N, et al. Gefitinib or carboplatin-paclitaxel in pulmonary adenocarcinoma. N Engl J Med 2009; 361: 947-57. doi:10.1056/NEJMoa0810699

3. Zhou C, Wu YL, Chen G, Feng J, Liu XQ, Wang C, et al. Final overall survival results from a randomised, phase III study of erlotinib versus chemotherapy as first-line treatment of EGFR mutation-positive advanced non-smallcell lung cancer (OPTIMAL, CTONG-0802). Ann Oncol 2015; 26: 1877-83. doi:10.1093/annonc/mdv276

4. Sequist LV, Yang JC, Yamamoto N, O'Byrne K, Hirsh V, Mok T, et al. Phase III study of afatinib or cisplatin plus pemetrexed in patients with metastatic lung adenocarcinoma with EGFR mutations. J Clin Oncol 2013; 31: 3327-34. doi:10.1200/JCO.2012.44.2806

5. Rosell R, Carcereny E, Gervais R, Vergnenegre A, Massuti B, Felip E, et al. Erlotinib versus standard chemotherapy as first-line treatment for European patients with advanced EGFR mutation-positive non-small-cell lung cancer (EURTAC): a multicentre, open-label, randomised phase 3 trial. Lancet Oncol 2012; 13: 239-46. doi:10.1016/S1470-2045(11)70393-X

6. Inoue A, Yoshida K, Morita S, Imamura F, Seto T, Okamoto I, et al. Characteristics and overall survival of EGFR mutation-positive non-small cell lung cancer treated with EGFR tyrosine kinase inhibitors: a retrospective analysis for 1660 Japanese patients. Jpn J Clin Oncol 2016; 46: 462-7. doi. org/10.1093/jjco/hyw014

7. Zhou C, Yao LD. Strategies to improve outcomes of patients with EGRFmutant non-small cell lung cancer: Review of the literature. J Thorac Oncol 2016; 11: 174-86. doi:10.1016/j.jtho.2015.10.002

8. Giaccone G, Herbst RS, Manegold C, Scagliotti G, Rosell R, Miller V, et al. Gefitinib in combination with gemcitabine and cisplatin in advanced nonsmall-cell lung cancer: a phase III trial - INTACT 1. J Clin Oncol 2004; 22: 777-84. doi:10.1200/JCO.2004.08.001

9. Herbst RS, Giaccone G, Schiller JH, Natale RB, Miller V, Manegold C, et al. Gefitinib in combination with paclitaxel and carboplatin in advanced nonsmall-cell lung cancer: a phase III trial - INTACT 2. J Clin Oncol 2004; 22: 785-94. doi:10.1200/JCO.2004.07.215

10. Herbst RS, Prager D, Herman R, Fehrenbacher L, Johnson BE, Sandler A, et al. TRIBUTE: a phase III trial of erlotinib hydrochloride (OSI-774) combined with carboplatin and paclitaxel chemotherapy in advanced non-small-cell lung cancer. J Clin Oncol 2005; 23: 5892-9. doi:10.1200/JCO.2005.02.840

11. Gatzemeier U, Pluzanska A, Szczesna A, Kaukel E, Roubec J, De Rosa F, et al. Phase III study of erlotinib in combination with cisplatin and gemcitabine in advanced non-small-cell lung cancer: The Tarceva Lung Cancer Investigation Trial. J Clin Oncol 2007; 25: 1545-52. doi:10.1200/JCO.2005.05.1474

12. Ratti M, Tomasello G. Emerging combination therapies to overcome resistance in EGFR-driven tumors. Anticancer Drugs 2014; 25: 127-39. doi:10.1097/CAD.0000000000000035

13. Steuer CE, Khuri FR, Ramalingam SS. The next generation of epiderma growth factor receptor tyrosine kinase inhibitors in the treatment of lung cancer. Cancer 2015; 121: E1-E6. doi:10.1002/cncr.29139

14. Jänne PA, Yang JC, Kim DW, Planchard D, Ohe $Y$, Ramalingam SS, et al AZD9291 in EGFR inhibitor-resistant non-small-cell lung cancer. N Engl J Med 2015; 372: 1689-99. doi:10.1056/NEJMoa1411817 
15. Eberhard DA, Johnson BE, Amler LC, Goddard AD, Heldens SL, Herbst RS, et al. Mutations in the epidermal growth factor receptor and in KRAS are predictive and prognostic indicators in patients with non-small-cell lung cancer treated with chemotherapy alone and in combination with erlotinib. J Clin Oncol 2005; 23: 5900-909. doi:10.1200/JCO.2005.02.857

16. Reck M. Beyond the TRIBUTE trial: integrating HER1/EGFR tyrosine kinase inhibitors with chemotherapy in advanced NSCLC. Future Oncol 2006; 2: 47-51. doi:10.2217/14796694.2.1.47

17. Tsai CM, Chen JT, Chiu CH, Lai CL, Hsiao SY, Chang KT. Combined epidermal growth factor receptor (EGFR)-tyrosine kinase inhibitor and chemotherapy in non-small-cell lung cancer: chemo-refractoriness of cells harboring sensitizing-EGFR mutations in the presence of gefitinib. Lung Cancer 2013; 82: 305-12. doi:10.1016/j.lungcan.2013.08.028

18. Davies AM, Ho C, Lara PN Jr, Mack P, Gumerlock PH, Gandara DR. Pharmacodynamic separation of epidermal growth factor receptor tyrosine kinase inhibitors and chemotherapy in non-small-cell lung cancer. Clin Lung Cancer 2006; 7: 385-8. doi:10.3816/CLC.2006.n.02

19. Mahaffey CM, Davies AM, Lara PN Jr, Pryde B, Holland W, Mack PC, et al. Schedule-dependent apoptosis in K-ras mutant non-small-cell lung cancer cell lines treated with docetaxel and erlotinib: rationale for pharmacodynamic separation. Clin Lung Cancer 2007; 8: 548-53. doi:10.3816/ clc.2007.n.041

20. Piperdi B, Ling Y-H, Kroog G, Perez-Soler R. Schedule-dependent interaction between epidermal growth factor inhibitors (EGFRI) and G2/M blocking chemotherapeutic agents (G2/MB) on human NSCLC cell lines in vitro. J Clin Oncol 2004; 22(14S): 7028. PMID: 28016090

21. Solit DB, She Y, Lobo J, Kris MC, Scher HI, Rosen N, et al. Pulsatile administration of epidermal growth factor receptor inhibitor gefitinib is significantly more effective than continuous dosing for sensitizing tumors to paclitaxel. Clin Cancer Res 2005; 11: 1983-9. doi:10.1158/1078-0432.CCR-04-1347

22. Bartholomeusz C, Yamasaki F, Saso H, Kurisu K, Hortobagyi GN, Ueno NT. Gemcitabine overcomes erlotinib resistance in EGFR-overexpressing cancer cells through downregulation of Akt. J Cancer 2011; 2: 435-42. doi:10.7150/ jca.2.435

23. Kanda S, Horinouchi H, Fujiwara Y, Nokihara H, Yamamoto N, Sekine I, et al. Cytotoxic chemotherapy may overcome the development of acquired resistance to epidermal growth factor receptor tyrosine kinase inhibitors (EGFR-TKIs) therapy. Lung Cancer 2015; 89: 287-93. doi:10.1016/j.lungcan.2015.06.016

24. La Salvia A, Rossi A, Galetta D, Gobbini E, De Luca E, Novello S, et al. Intercalated chemotherapy and epidermal growth factor receptor inhibitors for patients with advanced non-small-cell lung cancer: A systematic review and meta-analysis. Clin Lung Cancer 2017; 18: 23-33. doi:10.1016/j. cllc.2016.08.006

25. Midha A, Dearden S, McCormack R. EGFR mutation incidence in non-smal cell lung cancer with adenocarcinoma histology: a systematic review and global map by ethnicity (mutMaplI). Am J Cancer Res 2015; 5: 2892-11. PMID: 26609494

26. Mok TS, Wu YL, Yu CJ, Zhou C, Chen YM, Zhang L, et al. Randomized, placebo-controlled, phase II study of sequential erlotinib and chemotherapy as first-line treatment for advanced non-small-cell lung cancer. J Clin Oncol 2009; 27: 5080-7. doi:10.1200/JCO.2008.21.5541

27. Hirsch FR, Kabbinavar F, Eisen T, Martins R, Schnell FM, Dziadziuszko R, et al. A randomized, phase II, biomarker-selected study comparing erlotinib to erlotinib intercalated with chemotherapy in first-line therapy for advanced non-small-cell lung cancer. J Clin Oncol 2011; 29: 3567-73. doi:10.1200/ JCO.2010.34.4929

28. Aerts JG, Codrington H, Lankheet NAG, Burgers S, Biesma B, Dingemans AM, et al. A randomized phase II study comparing erlotinib versus erlotinib with alternating chemotherapy in relapsed non-small-cell lung cancer patients: The NVALT-10 study. Ann Oncol 2013; 24: 2860-5. doi:10.1093/annonc/ mdt341

29. Lee DH, Lee JS, Kim SW, Rodrigues-Pereira J, Han B, Song XQ, et al. Threearm randomised controlled phase 2 study comparing pemetrexed and erlotinib to either pemetrexed or erlotinib alone as second-line treatment for never-smokers with non-squamous non-small cell lung cancer. Eur J Cancer 2013; 49: 3111-21. doi:10.1016/j.ejca.2013.06.035

30. Wu YL, Lee JS, Thongprasert S, Yu CJ, Zhang L, Ladrera G, et al. Intercalated combination of chemotherapy and erlotinib for patients with advanced stage non-small-cell lung cancer (FASTACT 2): a randomised, double blind trial. Lancet Oncol 2013; 14: 777-86. doi:10.1016/S1470-2045(13)70254-7
31. Auliac JB, Chouaid C, Greillier L, Monnet I, Le Caer H, Falchero L, et al. Randomized open-label non-comparative multicenter phase II trial of sequential erlotinib and docetaxel versus docetaxel alone in patients with nonsmall-cell lung cancer after failure of first-line chemotherapy: GFPC 10.02 study. Lung Cancer 2014; 85: 415-9. doi:10.1016/j.lungcan.2014.07.006

32. Karavasilis V, Kosmidis P, Syrigos KN, Mavropoulou P, Dimopoulos MA Kotoula $\mathrm{V}$, et al. Docetaxel and intermittent erlotinib in patients with metastatic Non-Small Cell Lung Cancer; a phase II study from the Hellenic Cooperative Oncology Group. Anticancer Res 2014; 34: 5649-55. PMID: 25275069

33. Mok TS, Gaeter SL, lannotti N, Thongprasert S, Spira A, Smith D, et al. Randomized phase II study of two intercalated combinations of eribulin mesylate and erlotinib in patients with previously treated advanced nonsmall-cell lung cancer. Ann Oncol 2014; 25: 1578-84. doi:10.1093/annonc/ mdu174

34. Yu H, Zhang J, Wu X, Luo Z, Wang $\mathrm{H}$, Sun $\mathrm{S}$, et al. A phase II randomized trial evaluating gefitinib intercalated with pemetrexed/platinum chemotherapy or pemetrexed/platinum chemotherapy alone in unselected patients with advanced non-squamous non-small cell lung cancer. Cancer Biol Ther 2014; 15: 832-9. doi:10.4161/cbt.28874

35. Choi YJ, Lee DH, Choi CM, Lee JS, Lee SJ, Ahn JH, et al. Randomized phase II study of paclitaxel/carboplatin intercalated with gefitinib compared to paclitaxel/carboplatin alone for chemotherapy-naïve non-small cell lung cancer in a clinically selected population excluding patients with non-smoking adenocarcinoma or mutated EGFR. BMC Cancer 2015; 15: 763. doi:10.1186/ s12885-015-1714y

36. Juan Ó, Aparisi F, Sánchez-Hernández A, Muñoz-Langa J, Esquerdo G, GarcíaSánchez J, et al. Intercalated dosing schedule of erlotinib and docetaxel as a therapeutic strategy to avoid antagonism and optimize its benefits in advanced non-small-cell lung cancer. A randomized phase II clinical trial. Clin Lung Cancer 2015; 16: 193-9. doi:10.1016/j.cllc.2014.11.006

37. Lu S, Jian H, Li W, Yong MZ, Huang JJ, Feng J, et al. Intercalating and maintenance use of gefitinib plus chemotherapy versus chemotherapy alone in selected advanced NSCLC (ISCAN,CTONG-1102): a multicentre, open-label, randomised, phase 3 study. [abstract]. J Clin Oncol 2015; 33(Suppl): abstr 8042. ClinicalTrials.gov Identifier: NCT01404260

38. Michael M, White SC, Abdi E, Nott L, Clingan P, Zimet A, et al. Multicenter randomized, open-label phase II trial of sequential erlotinib and gemcitabine compared with gemcitabine monotherapy as first-line therapy in elderly or ECOG PS two patients with advanced NSCLC. Asia Pac J Clin Oncol 2015; 11: 4-14. doi:10.1111/ajco.12178

39. Han B, Zhang Y, Jin B, Chu T, Gu A, Xu J. Combination of chemotherapy and gefitinib as first-line treatment of patients with advanced lung adenocarcinoma and sensitive EGFR mutations: a randomized controlled trial. J Thorac Oncol 2016; 11(Suppl 4): S113-4. doi:10.1016/S1556-0864(16)30244-1

40. Lara PN Jr, Moon J, Hesketh PJ, Redman MW, Williamson SK, Akerley WL 3rd, et al. SWOG S0709: Randomized Phase II trial of erlotinib versus erlotinib plus carboplatin/paclitaxel in patients with advanced non-small cell lung cancer and impaired performance status as selected by a serum proteomics assay. J Thorac Oncol 2016; 11: 420-5. doi:10.1016/j.jtho.2015.11.003

41. Li T, Piperdi B, Walsh WV, Kim M, Beckett A, Gucalp R, et al. Randomized phase II study of pharmacodynamic separation of pemetrexed and intercalated erlotinib versus pemetrexed alone for advanced non-small cell lung cancer. Clin Lung Cancer 2017; 18: 60-7. doi 10.1016/j.cllc.2016.10.003

42. Lee JS, Lee YJ, Kim HY, Nam B-H, Lee GK, Kim HT, et al. Randomized phase II trial of intercalated gefitinib (G) and pemetrexed/cisplatin (Pem/Cis) for never-smokers with chemo-naïve stage IIIB/IV lung adenocarcinoma (LADC). [abstract]. J Clin Oncol 2016; 34: e20505. ClinicalTrials.gov Identifier: NCT01502202

43. Yoon S, Lee D-H, Kim D, Choi C-M, Kim S-W. Randomized Phase II tria comparing intercalation of afatinib to pemetrexed with pemetrexed alone after failure of platinum doublet therapy. [abstract] J Thorac Oncol 2017; 12(Suppl 1): S926. abstr P2.03A-061

44. Oshita F, Saito H, Murakami S, Kondo T, Yamada K. Phase II study of paclitaxel and irinotecan with intercalated gefitinib in patients with advanced non-small-cell lung cancer. Am J Clin Oncol 2010; 33: 66-9. doi:10.1097/ cOC.0b013e31819ccc6d

45. Sangha R, Davies AM, Lara PN Jr, Mack PC, Beckett LA, Hesketh PJ, et al Intercalated erlotinib-docetaxel dosing schedules designed to achieve pharmacodynamic separation: results of a phase I/II trial. J Thorac Oncol 2011. 6: 2112-9. doi:10.1097/JTO.0b013e31822ae061 
46. Minami S, Kijima T, Hamaguchi M, Nakatani T, Koba T, Takahashi R, et al. Phase II study of pemetrexed plus intermittent erlotinib combination therapy for pretreated advanced non-squamous non-small cell lung cancer with documentation of epidermal growth factor receptor mutation status. Lung Cancer 2013; 82: 271-5. doi:10.1016/j.lungcan.2013.07.022

47. Yoshimura N, Okishio K, Mitsuoka S, Kimura T, Kawaguchi T, Kobayashi M, et al. Prospective assessment of continuation of erlotinib or gefitinib in patients with acquired resistance to erlotinib or gefitinib followed by the addition of pemetrexed. J Thorac Oncol 2013; 8: 96-101. doi:10.1097/ JTO.0b013e3182762bfb

48. Kim YH, Nishimura T, Ozasa H, Nagai H, Sakamori Y, Iwata T, et al. Phase II study of pemetrexed and erlotinib in pretreated nonsquamous non-smallcell lung cancer patients without an EGFR mutation. Chemotherapy 2013; 59: 414-9. doi:10.1159/000363731

49. Fang $H$, Lin $R Y$, Sun $M X$, Wang $Q$, Zhao $Y L$, Yu JL, et al. Efficacy and survivalassociated factors with gefitinib combined with cisplatin and gemcitabine for advanced non- small cell lung cancer. Asian Pac J Cancer Prev 2014;15: 10967-70. doi:10.7314/APJCP.2014.15.24.10967

50. Yang J, Shi Y, Zhang X, Xu J, Wang B, Hao X et al. Phase II trial of paclitaxelcarboplatin with intercalated gefitinib for untreated, epidermal growth factor receptor gene mutation status unknown non-small cell lung cancer. Thorac Cancer 2014; 5: 149-54. doi:10.1111/1759-7714.12074

51. Zwitter M, Stanič K, Rajer M, Kern I, Vrankar M, Edelbaher N, et al. Intercalated chemotherapy and erlotinib for advanced NSCLC: high proportion of complete remissions and prolonged progression-free survival among patients with EGFR activating mutations. Radiol Oncol 2014; 48: 361-8. doi:10.2478/raon-2014-0038

52. Yoshimura $\mathrm{N}$, Kudoh $\mathrm{S}$, Mitsuoka $\mathrm{S}$, Yoshimoto $\mathrm{N}$, Oka $\mathrm{T}$, Nakai $\mathrm{T}$, et al. Phase II study of a combination regimen of gefitinib and pemetrexed as first-line treatment in patients with non-small cell lung cancer harbouring a sensitive EGFR mutation. Lung Cancer 2015; 90: 65-70. doi:10.1016/j. lungcan.2015.06.002

53. Yu S, Zhang B, Xiang C, Shu Y, Wu H, Huang X, et al. Prospective assessment of pemetrexed or pemetrexed plus platinum in combination with gefitinib or erlotinib in patients with acquired resistance to gefitinib or erlotinib: a phase II exploratory and preliminary study. Clin Lung Cancer 2015; 16: 12127. doi:10.1016/j.cllc.2014.09.007

54. Zwitter M, Rajer M, Stanic K, Vrankar M, Doma A, Cuderman A, et al. Intercalated chemotherapy and erlotinib for non-small cell lung cancer (NSCLC) with activating epidermal growth factor receptor (EGFR) mutations. Cancer Biol Ther 2016; 17: 833-9. doi:10.1080/15384047.2016.1195049

55. Chen YM, Lai CH, Chang HC, Chao TY, Tseng CC, Fang WF, et al. The impact of clinical parameters on progression-free survival of non-small cell lung cancer patients harboring EGFR-mutations receiving first-line EGFRtyrosine kinase inhibitors. Lung Cancer 2016; 93: 47-54. doi:10.1016/j. lungcan.2016.01.001

56. De Grève J, Van Meerbeeck J, Vansteenkiste JF, Decoster L, Meert AP, Vuylsteke $P$, et al. Prospective evaluation of first-line erlotinib in advanced non-small cell lung cancer (NSCLC) carrying an activating EGFR mutation: A multicenter academic phase II study in Caucasian patients (FIELT). PLoS One 2016; 11: e0147599. doi:10.1371/journal.pone.0147599

57. Douillard JY, Ostoros G, Cobo M, Ciuleanu T, McCormack R, Webster A, et al. First-line gefitinib in Caucasian EGFR mutation-positive NSCLC patients: a phase-IV, open-label, single-arm study. Br J Cancer 2014; 110: 55-62. doi:10.1038/bjc.2013.721

58. Inoue A, Yoshida K, Morita S, Imamura F, Seto T, Okamoto I, et al. Characteristics and overall survival of EGFR mutation-positive non-small cell lung cancer treated with EGFR tyrosine kinase inhibitors: a retrospective analysis for 1660 Japanese patients. Jpn J Clin Oncol 2016; 46: 462-7. doi:10.1093/jjco/hyw014

59. Rossi A. Chemotherapy plus intercalated or continuous EGFR-TKI in advanced non-small cell lung cancer. Transl Cancer Res 2016; 5(Suppl 4): S659-63.

60. Lopes G, Tan PS, Acharyya S, Bilger M, Haaland B. Network meta-analysis comparing first-line therapies and maintenance regimens in EGFR mutated advanced non-small-cell lung cancer (NSCLC). [abstract]. J Clin Oncol 2016; 34(Suppl): abstr e20570.

61. Sacher AG, Komatsubara KM, Oxnard GR. Application of plasma genotyping technologies in non-small cell lung cancer: a practical review. J Thorac Oncol 2017; [Epub ahead of print]. doi:10.1016/j.jtho.2017.05.022
62. Mok TS, Wu Y-L, Ahn M-J, Garassino MC, Kim HR, Ramalingam SS, et al. Osmertinib or platinum-pemetrexed in EGFR T790M-positive lung cancer. $N$ Engl J Med 2016; 376: 629-40. doi:10.1056NEJMMoa1612674

63. Sugawara $S$, Oizumi $S$, Minato $K$, Harada $T$, Inoue A, Fujita $Y$, et al. Randomized phase II study of concurrent versus sequential alternating gefitinib and chemotherapy in previously untreated non-small cell lung cancer with sensitive EGFR mutations: NEJ005/TCOG0902. Ann Oncol 2015; 26: 888-94. doi:10.1093/annonc/mdv063

64. Cheng Y, Murakami H, Yang PC, He J, Nakagawa K, Kang JH, et al. Randomized Phase II trial of gefitinib with and without pemetrexed as firstline therapy in patients with advanced nonsquamous non-small-cell lung cancer with activating epidermal growth factor receptor mutations. J Clin Oncol 2016; 34: 3258-66. doi:10.1200/JCO.2016.66.9218

65. Seto T, Kato T, Nishio M, Goto K, Atagi S, Hosomi Y, et al. Erlotinib alone or with bevacizumab as first-line therapy in patients with advanced non-squamous non-small-cell lung cancer harbouring EGFR mutations (JO25567): an open-label, randomised, multicentre, phase 2 study. Lancet Oncol 2014; 15 : 1236-44. doi:10.1016/S1470-2045(14)70381-X 